\title{
Searches for resonant and non-resonant new phenomena in ATLAS
}

\section{Wojciech T. Fedorko* on behalf of ATLAS Collaboration}

University of British Columbia, Vancouver, BC, Canada

E-mail: wfedorko@phas.ubc.ca

\begin{abstract}
Many new physics scenarios beyond the Standard Model predict the presence of narrow or broad resonances decaying to a pair of quarks/gluons, charged/neutral leptons, photons and their combinations, or a multi-jet final state. Non-resonant excess in tails of mass and transverse momentum distributions is another strong indication of new physics. In these proceedings highlights of recent ATLAS searches on new phenomena in dijet, multi-jet, dilepton, photon+jet and diphoton final states using LHC Run 1 data are presented. First results using LHC Run 2 data are also shown.
\end{abstract}

The European Physical Society Conference on High Energy Physics 22-29 July 2015

Vienna, Austria

${ }^{*}$ Speaker. 


\section{Introduction}

In these proceedings we present the state of a subset of ATLAS [1] searches for physics beyond the Standard Model as of Summer 2015. Searches presented here use full $\sqrt{s}=8 \mathrm{TeV}$ dataset from LHC Run 1. In addition first results from ATLAS using Run 2 dataset are shown in section 9. A variety of searches encompassing exotics sector are presented. These include searches for new heavy gauge bosons, gravitons, contact interactions, extra dimensions, semi-classical and quantum black holes, excited quarks and leptoquarks.

\section{Searches in the dilepton final states}

The final state with two electrons or two muons is sensitive to a number of new physics scenarios. Two analysis are performed in the dilepton channel. One is a search for new physics with a resonant final state [2]. In the second paper non-resonant final states are explored [3]. For both analysis two high momentum electrons or muons are selected. Quality criteria based on shower shape and track quality are applied to select the electron candidates. Muon candidates are selected based on inner detector quality cuts and muon spectrometer quality cuts tuned for very high transverse momenta. The two muon candidates are required to have opposite charge. Two electron candidates are required to have opposite charge only in the search for non-resonant final states. Figure 1 shows distributions of dilepton masses in the dielectron channel (a) and in the dimuon channel (b). No excess over the Standard Model (SM) prediction is observed in either channel. Data from both channels is used simultaneously to set a cross section times branching ratio $(\sigma B)$ limits for production of a variety of new physics models including the Sequential Standard Model (SSM) $Z^{\prime}$ [4], the $E(6) Z^{\prime}$ [5] and the Randall-Sundrum (RS) graviton excitation $G^{*}$ [6] taking into account the systematic uncertainties. The SSM $Z^{\prime} \sigma B$ 95\% confidence level (CL) limits are shown in figure 2a. The observed (expected) lower pole mass limit for the SSM $Z^{\prime}$ is $2.90 \mathrm{TeV}$ (2.87 $\mathrm{TeV}$ ). For the $E(6) Z^{\prime}$ the observed pole mass limits vary between 2.62 and $2.85 \mathrm{TeV}$ depending on the model. For the graviton excitation observed (expected) lower mass limit varies from 1.25 (1.28) $\mathrm{TeV}$ to 3.05 (3.05) $\mathrm{TeV}$ for values of the coupling parameter $k / \bar{M}_{\mathrm{Pl}}$ between 0.01 and 0.2 . Coupling limits are also set in the Minimal $Z^{\prime}$ models [7] as shown in Fig. 2b. In the non-resonant search [3], where angular distributions of the lepton pair used in conjunction with the invariant mass distribution lower limits are set on the contact interaction scale $\Lambda[8,9]$ between 15.4 to $26.3 \mathrm{TeV}$ depending on the nature of the interaction and Bayesian prior chosen. In the context of Arkani-Hamed, Dimopoulos, Dvali (ADD) model [10] lower limits are set on the string scale $M_{\mathrm{S}}$ between 3.2 and $5.0 \mathrm{TeV}$.

A search for high-mass resonances is also performed in the $\tau^{+} \tau^{-}$final state [25]. Tau lepton candidates where the $\tau$ decays hadronically are identified using boosted decision trees to discriminate agaist quark- and gluon-initiated jets using shower shape and tracking information. One and three prong hadronic tau candidates $\tau_{\text {had }}$ are used. Two channels are used: one with both taus decaying hadronically ( $\tau_{\text {had }} \tau_{\text {had }}$ ) and one where one of the taus decays semileptonically into an isolated electron or muon $\left(\tau_{\text {lep }} \tau_{\text {had }}\right)$. In both channels a requirement is made that the two objects are back-toback with azimuthal angle difference ${ }^{1} \Delta \phi>2.7$ radians. The transverse mass of two objects is de-

\footnotetext{
${ }^{1}$ ATLAS uses a right-handed coordinate system with its origin at the nominal interaction point (IP) in the centre
} 


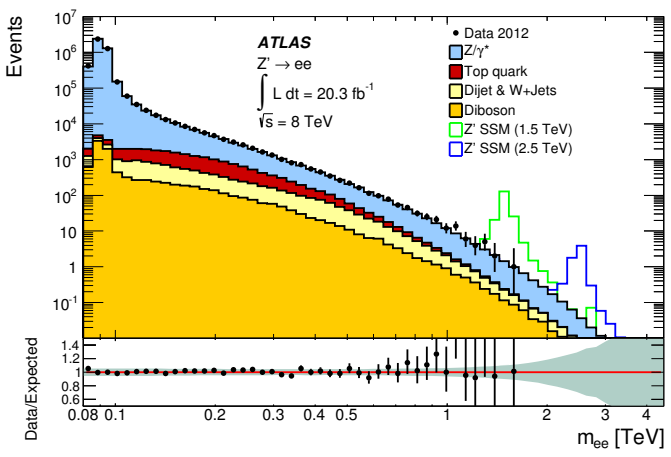

(a)

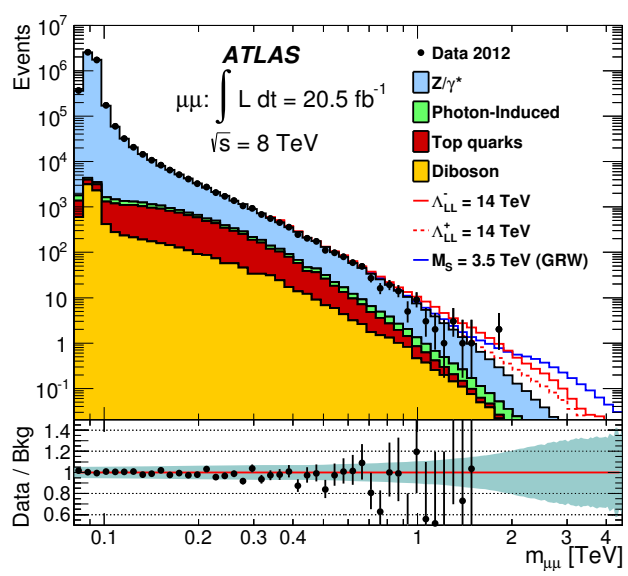

(b)

Figure 1: Invariant mass of two-lepton system: in the dielectron channel (a) with SSM Z' signal shapes overlaid from [2] and in dimuon channel (b) with contact interaction signals overlaid from [3]

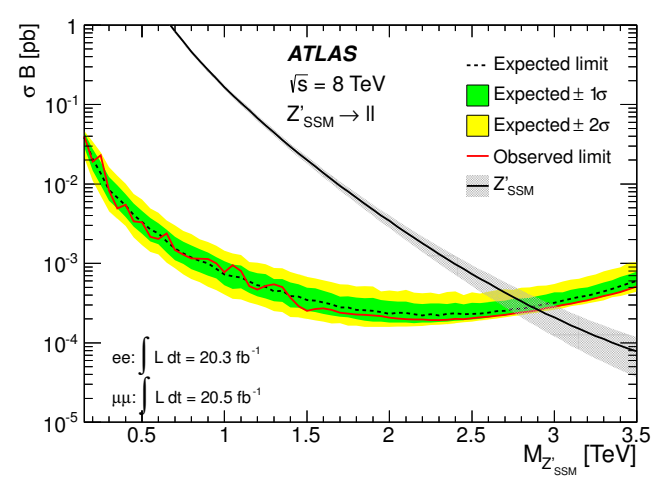

(a)

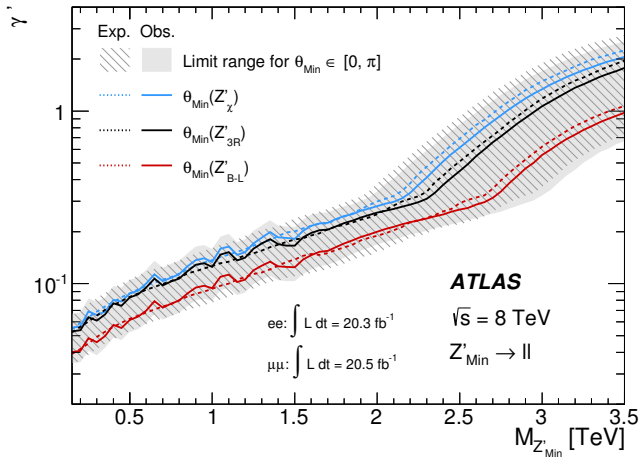

(b)

Figure 2: 95\% CL upper limits on the $\sigma B$ for SSM $Z$ ' production (a) and 95\% CL upper limits on the strength of the $Z$ boson coupling relative to that of the SM Z boson $\left(\gamma^{\prime}\right)$ for the combined dielectron and dimuon channels as a function of the $Z^{\prime}$ pole mass in the Minimal $Z$ Models parameterization (b). Both diagrams from [2]

fined as $m_{\mathrm{T}}\left(p^{\mathrm{A}}, p^{\mathrm{B}}\right)=\sqrt{2 p_{\mathrm{T}}^{\mathrm{A}} p_{\mathrm{T}}^{\mathrm{B}}\left(1-\cos \Delta \phi\left(p^{\mathrm{A}}, p^{\mathrm{B}}\right)\right)}$. In the $\tau_{\text {lep }} \tau_{\text {had }}$ channel $m_{\mathrm{T}}\left(l, E_{\mathrm{T}}^{\text {miss }}\right)$ must be less than $50 \mathrm{GeV}$ to suppress contributions from $W$ decay. Counting expetiments are performed in samples with a threshold on total transverse mass $m_{\mathrm{T}}^{\text {tot }}=\sqrt{m_{\mathrm{T}}^{2}\left(\tau_{1}, \tau_{2}\right)+m_{\mathrm{T}}^{2}\left(\tau_{1}, E_{\mathrm{T}}^{\text {miss }}\right)+m_{\mathrm{T}}^{2}\left(\tau_{2}, E_{\mathrm{T}}^{\text {miss }}\right)}$ where $\tau_{1}$ and $\tau_{2}$ denote the reconstructed visible decay products of the two tau leptons. The $m_{\mathrm{T}}^{\text {tot }}$ thresholds are optimised based on sensitivity to particular signal mass hypothesis. The distribution

of the detector and the $z$-axis along the beam pipe. The $x$-axis points from the IP to the centre of the LHC ring, and the $y$-axis points upwards. Cylindrical coordinates $(r, \phi)$ are used in the transverse plane, $\phi$ being the azimuthal angle around the $z$-axis. The pseudorapidity is defined in terms of the polar angle $\theta$ as $\eta=-\ln \tan (\theta / 2)$. Angular distance is measured in units of $\Delta R \equiv \sqrt{(\Delta \eta)^{2}+(\Delta \phi)^{2}}$. 
of $m_{\mathrm{T}}^{\text {tot }}$ in $\tau_{\text {had }} \tau_{\text {had }}$ and $\tau_{\text {lep }} \tau_{\text {had }}$ channels is shown in 3. No excess is found over the SM expectation and upper limits on $Z^{\prime}$ production cross section times branching ratio are set excluding SSM $Z^{\prime}$ below $2.02 \mathrm{TeV}$.

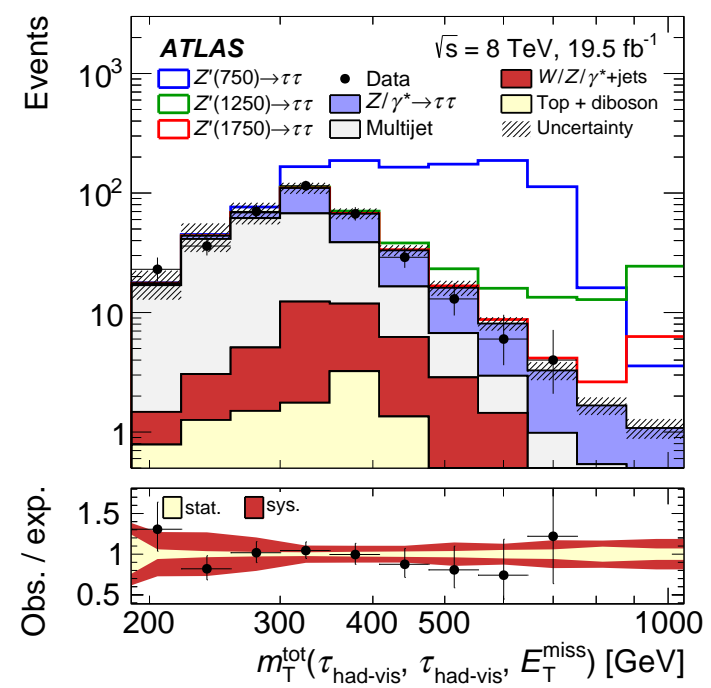

(a)

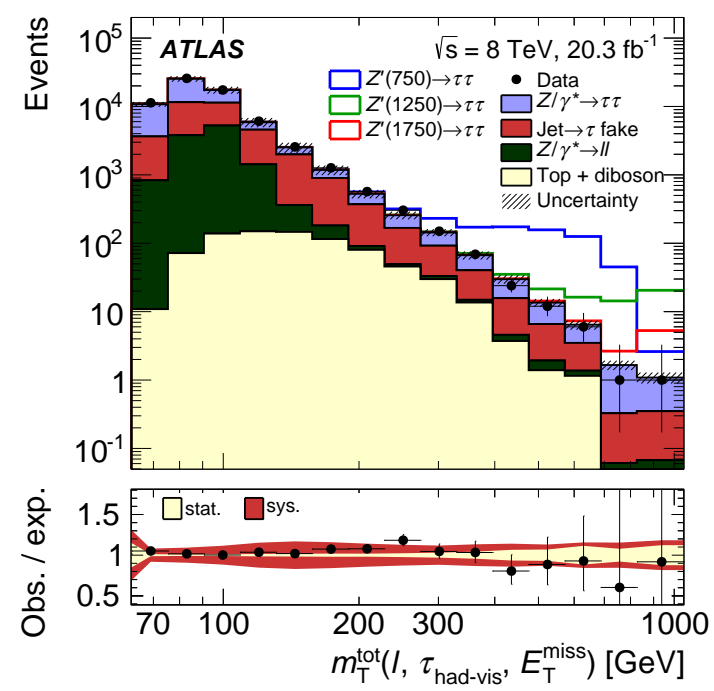

(b)

Figure 3: Total transverse mass distribution in the $\tau_{\text {had }} \tau_{\text {had }}$ (a) and $\tau_{\text {lep }} \tau_{\text {had }}$ (b) channels. Both diagrams from [25].

\section{Diphoton resonance search}

In this search [11] two photon candidates (including photon conversions) are selected based on calorimetric shower shape variables such as the energy leakage into the hadronic calorimeter and the width of the shower in the first and second layers of the electromagnetic calorimeter. The Standard Model backgrounds are estimated using Monte Carlo (MC) simulations for the irreducible backgrounds and a data driven method for the events where one or two jets are reconstructed as photons. No excess is found in the diphoton invariant mass distribution shown in Fig. 4a. Using the full mass shape upper limits are set on the RS graviton production cross section times branching ratio to two photons (shown in Fig. 4b), which are interpreted as lower limits on the $G^{*}$ mass between 1.41 and $2.66 \mathrm{TeV}$ for $k / \bar{M}_{\mathrm{Pl}}$ values between 0.01 and 0.1 .

\section{Search for new particles in events with one lepton and missing transverse momentum}

Additional heavy gauge bosons may be produced at the LHC. A new physics scenario considered in this study [12] is the production of a SSM $W^{\prime}$ boson [13] with the $W^{\prime}$ decaying into a lepton-neutrino final state. Only electron and muon final states have been considered in this study. Electron candidates with transverse energy grater than $125 \mathrm{GeV}$ are selected based on tracking quality criteria and calorimetric shower shapes. Muon candidates with transverse momentum 


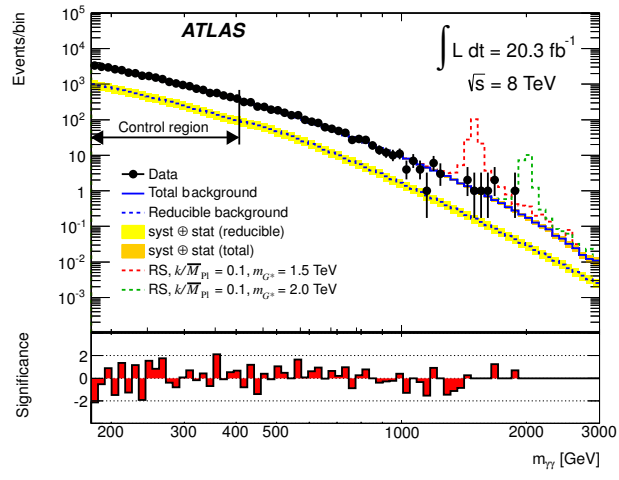

(a)

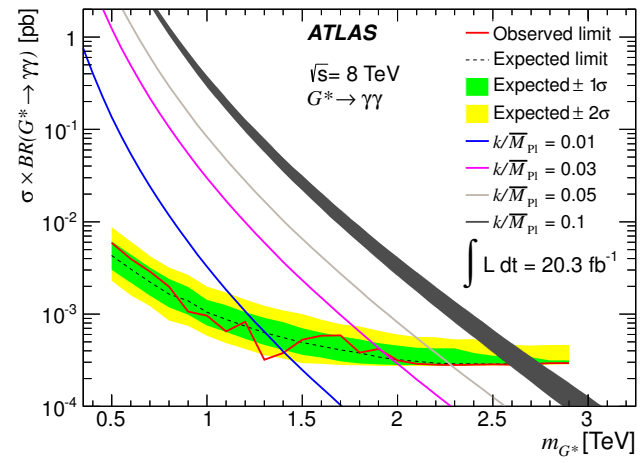

(b)

Figure 4: Invariant mass of the two photon system with RS graviton signals overlaid (a) and upper limits on $\mathrm{RS} G^{*}$ production cross section timed branching ratio. Both diagrams from [11].

greater than $45 \mathrm{GeV}$ are selected based on inner detector and muon spectrometer track quality criteria tuned for leptons with very high momenta. Both electron and muon candidates are required to be isolated. Calorimetric isolation criteria are used for the electrons and track isolation criteria are used for muons. Events with more than one lepton candidates are rejected. The missing transverse energy $E_{\mathrm{T}}^{\mathrm{miss}}$ in each event is evaluated by summing over energy-calibrated physics objects (jets, photons and leptons) and adding corrections for calorimeter deposits not associated with these objects. In the events with an electron (muon) candidate $E_{\mathrm{T}}^{\text {miss }}$ is required to be greater than $125 \mathrm{GeV}$ $(45 \mathrm{GeV})$. Background from event with a jet faking a lepton are evaluated using a combination of data-driven methods. Other backgrounds are estimated using MC simulated samples. The transverse mass $m_{\mathrm{T}}=\sqrt{2 p_{\mathrm{T}} E_{\mathrm{T}}^{\text {miss }}\left(1-\cos \varphi_{1 v}\right)}$ of the lepton- $E_{\mathrm{T}}^{\text {miss }}$ system is shown in Fig. 5, where $p_{\mathrm{T}}$ is the transverse momentum of the lepton and $\varphi_{1 v}$ is the angle between the $p_{\mathrm{T}}$ and $E_{\mathrm{T}}^{\text {miss }}$ vectors. No excess over the SM expectation is observed and upper limits are set on the SSM $W^{\prime} \sigma B$ using subsamples above a $m_{\mathrm{T}}$ threshold selected depending on the $W^{\prime}$ mass considered. SSM $W^{\prime}$ with mass below $3.24 \mathrm{TeV}$ is excluded at 95\% CL.

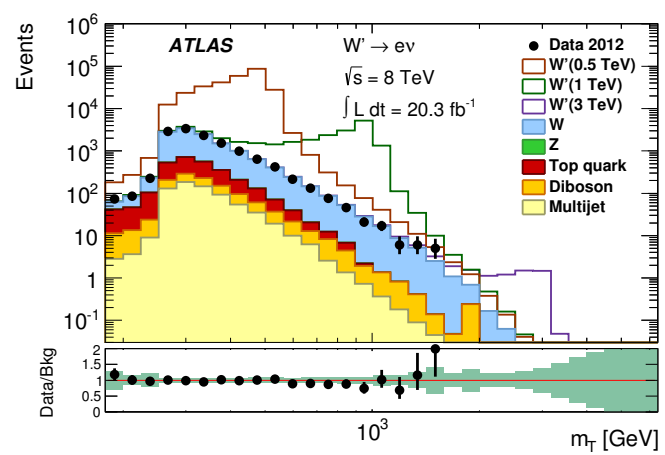

(a)

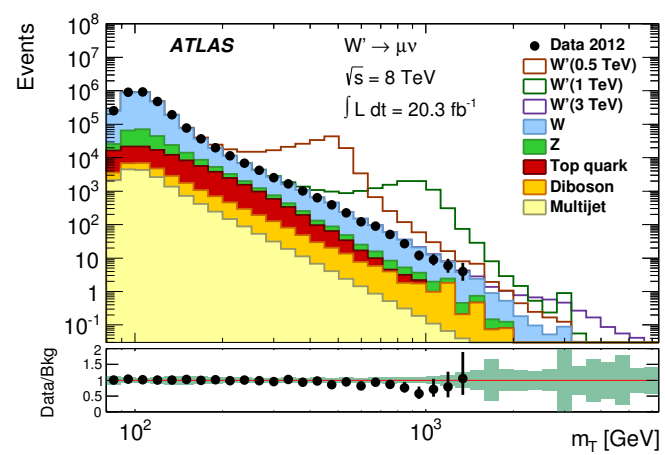

(b)

Figure 5: Transverse mass of the lepton- $E_{\mathrm{T}}^{\mathrm{miss}}$ system in the electron channel (a) and in the muon channel (b). Both diagrams from [12]. 


\section{Searches in dijet final states}

Two analyses of the dijet final states have been performed. The first [14] utilises dijet mass distribution and the second one [15] exploits dijet angular distributions. In both searches events with two jets with transverse momenta greater than $50 \mathrm{GeV}$ are selected. In the invariant mass search it is also required that the jet falls within the rapidity region of $|y|<2.8$. Events must also satisfy $\left|y^{*}\right|=\frac{1}{2}\left|y_{1}-y_{2}\right|<0.6$ in the mass search and $\left|y^{*}\right|<1.7$ in the angular search, where $y_{1}$ and $y_{2}$ are the rapidities of leading and sub-leading jets. In the angular search a requirement is also made on the boost of the dijet system defined as $y_{B}=\frac{1}{2}\left(y_{1}+y_{2}\right)<1.1$ and on the dijet mass $m_{\mathrm{jj}}>600 \mathrm{GeV}$. The mass search analysis makes a looser requirement on the invariant mass of the dijet system: $m_{\mathrm{jj}}>250 \mathrm{GeV}$.

In the mass search analysis an assumption of a smoothly falling Standard Model background is made. A smooth function is fitted to the data and resonant excesses are searched for above this background model. The distribution is shown in Fig. 6a. Given lack of any such excess upper limits on a number of new physics scenarios are set. In particular excited quarks $q^{*}[16,17]$ with masses below 4.06 $\mathrm{TeV}$ are excluded as are quantum black holes $[18,19,20,21]$ with six extra space-time dimensions with threshold mass $M_{\mathrm{TH}}$ below $5.66 \mathrm{TeV}$. Production cross section times acceptance upper limits are also set on generic Gaussian and Breit-Wigner resonances.

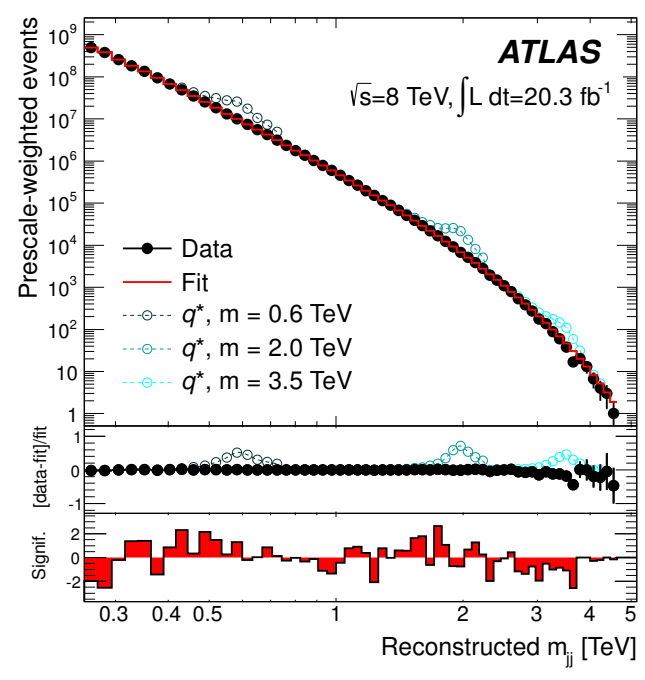

(a)

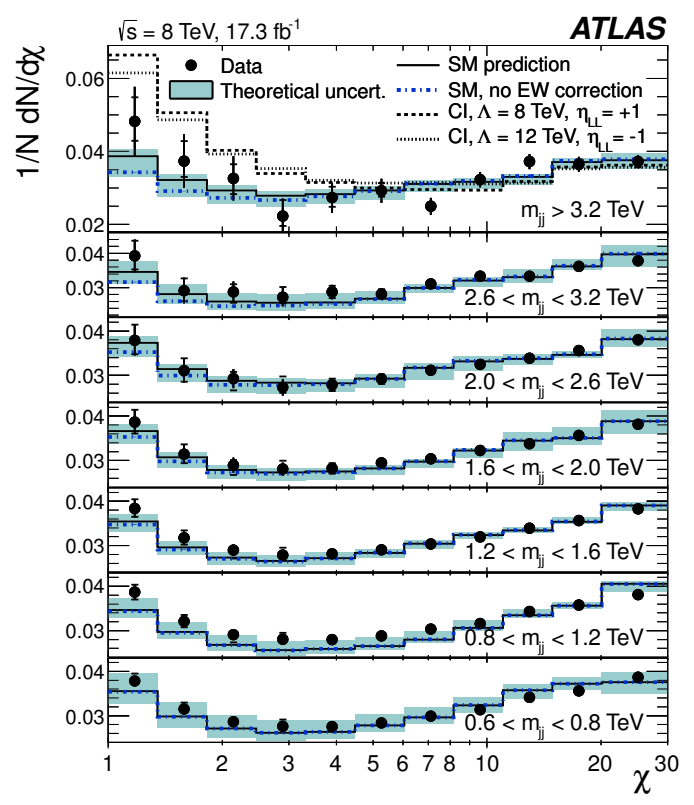

(b)

Figure 6: Invariant mass distribution of the dijet system with excited quark signals overlaid from [14] (a) and the dijet scattering angle $\chi$ distributions with contact interaction signals overlaid from [15] (b).

The angular search utilizes the dijet scattering angle $\chi=e^{\left|y^{*}\right|}$. The $\chi$ distribution is shown for several windows of $m_{\mathrm{jj}}$ in Fig. 6b. In each window the shape of the distribution is modeled by MC simulations however the normalisation is taken from the data. No significant deviation is observed from the Standard Model prediction and a lower limit on the interaction scale $\Lambda$ are set at $8.1 \mathrm{TeV}$ in 
the descructive interference scenario and $12.0 \mathrm{TeV}$ in a constructive interference scenario assuming only left-chiral coupling is present.

\section{Search for new phenomena in photon+jet events}

In this analysis [22] events with one photon and one jets each with $p_{\mathrm{T}}>125 \mathrm{GeV}$ are selected. The photon candidate is required to satisfy calorimetric isolation criteria and is also required to be seaprated from any jets with $p_{\mathrm{T}}>30 \mathrm{GeV}$. The separation has to be $\Delta R=<1.0$, where $\Delta \eta$ is pseudorapidity separation between photon and jet and $\Delta \phi$ is the asimuthal angle separation. Photon is required to be within the calorimeter barrel and photon jet pseudorapidity separation $\Delta \eta$ must be less than 1.6. The Standard Model background is assumed to be a smoothly decaying function and is estimated by a fit to the data (see Fig. 7a). In the absence of resonant exces over the SM background $95 \%$ CL limits are set on visible cross section for quantum black holes with six extra space-time dimensions and excited quarks. The visible cross section is the production cross section times brancing ratio times acceptance times efficiency $(\sigma \times B R \times A \times \varepsilon)$ where the acceptance $A$ is the fraction of events where the final state particles fall in the kinematic region where the detector is sensitive and $\varepsilon$ denotes the efficiency for the event within that region to satisfy selection criteria. Quantum black holes are excluded below masses of $4.6 \mathrm{TeV}$ and excited quarks are excluded below masses of 3.5 TeV. In addition $\sigma \times B R \times A \times \varepsilon$ upper limits are set for generic Gaussian-shaped resonances (Fig. 7b).

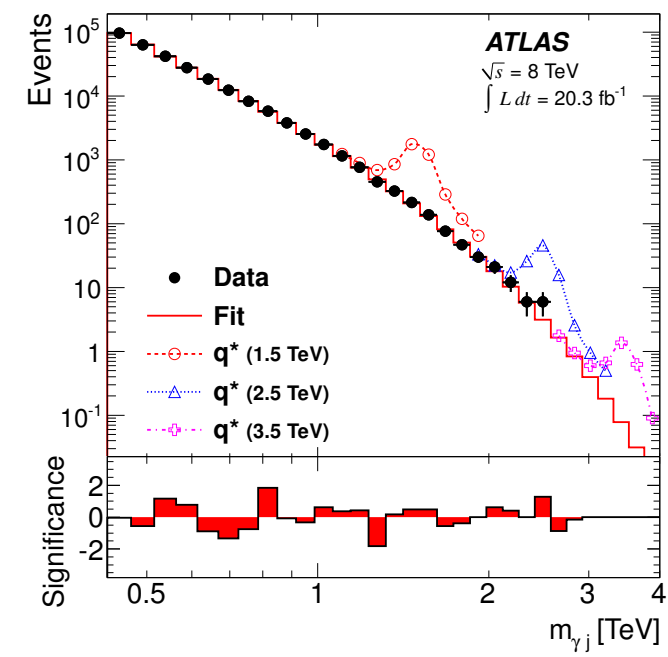

(a)

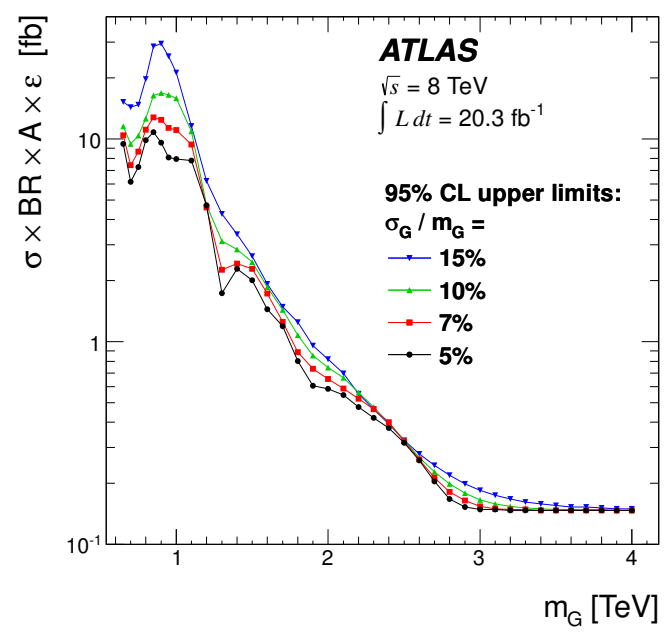

(b)

Figure 7: Invariant mass distribution of the $\gamma$-jet system with excited quark signals overlaid (a) and the 95\% CL upper limits on the visible cross section for generic Gaussian resonances (b). Both diagrams from [22].

\section{Search for low-scale gravity signatures in multi-jet final states}

In the context of low-scale gravity models with extra dimensions [10] black holes may be pro- 
duced at the LHC. If they are produced with masses much higher than the fundamental gravitational scale $M_{D}$ (the diminished effective Planck scale) they will decay to high multiplicity final states according to the SM degrees of freedom. Semi-classical approximations used in the modelling are valid for masses only well above $M_{D}$, motivating the use of a minimal threshold $M_{\text {th }}$ to remove contributions where the modelling is not reliable. Other high multiplicity final states such as string balls [23] may also be produced. In this analysis [24] final states where only jets are present are considered. All jets are required to have $p_{\mathrm{T}}>50 \mathrm{GeV}$. Linear sum of jet transverse momenta - the $H_{\mathrm{T}}$ is required to be greater than $1.5 \mathrm{TeV}$. Data with $H_{\mathrm{T}}<2.9 \mathrm{TeV}$ is defined as the control region used for fitting and extrapolating the $H_{\mathrm{T}}$ shape into the signal region with $H_{\mathrm{T}}>3.0 \mathrm{TeV}$. The search is divided into six overlapping regions of inclusive jet multiplicity: $N_{\text {jet }} \geq 3$ to $N_{\text {jet }} \geq 8$. The $H_{\mathrm{T}}$ distribution in the inclusive bin $N_{\text {jet }} \geq 3$ is shown in Fig. 8a. No significant excess is observed in any of the signal regions. Counting experiments with $H_{\mathrm{T}}>H_{\mathrm{T}}^{\min }$ as a function of $H_{\mathrm{T}}^{\min }$ are performed to set 95\% CL limits on the visible cross section. These limits are interpreted as exclusion limits on black holes in the $M_{D}-M_{\text {th }}$ plane and string balls in $M_{S}-M_{\text {th }}$ plane (where $M_{S}$ is the string ball mass). Selection of the $\left(N_{\mathrm{jet}}, H_{\mathrm{T}}^{\min }\right)$ point to constrain a given $\left(M_{S}, M_{\mathrm{th}}\right)$ or $\left(M_{D}, M_{\mathrm{th}}\right)$ is based on expected minimal signal p-value. Examples of the exclusion plots for selected model parameters are shown in Figures $8 b$ and $8 c$.

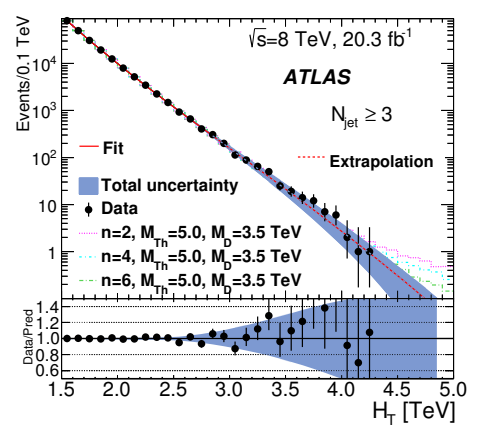

(a)

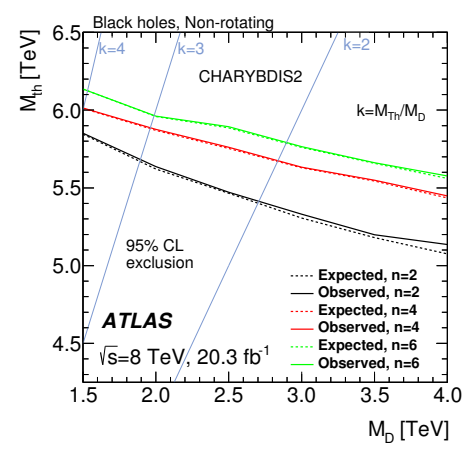

(b)

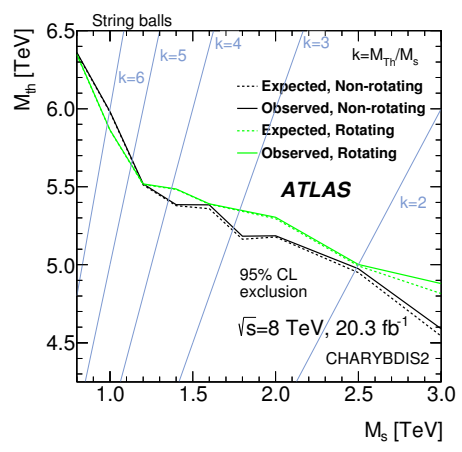

(c)

Figure 8: Distribution of $H_{\mathrm{T}}$ in the $N_{\text {jet }} \geq 3$ inclusive bin (a), non rotating semi-classical black hole exclusion plane (b) and the string ball exclusion plane. All diagrams from [24]

\section{Searches for scalar leptoquarks}

At the time of the conference a preliminary result was presented that has now been superseded by the final result described in [26]. In this analysis a search is performed for first and second generation leptoquarks [27]. Events with two isolated electrons or two isolated muons and two jets are selected. The muons are required to be opposite charge. Signal regions, optimized for signal significance, are constructed based on the invariant mass of the two leptons $m_{\mathrm{ll}}$, the scalar sum of the two leptons $S_{\mathrm{T}}$ and the lowest reconstructed leptoquark mass $m_{\mathrm{LQ}}^{\min }$ - picked from the two leptonpair combination that gives the smallest difference between the invariant masses of the lepton-jet pairings. The invariant mass of two electrons is shown in Fig. 9a and the $m_{\mathrm{LQ}}^{\min }$ in the muon channel 
is shown in Fig. 9b. No excess is observed in any of the signal regions thus excluding first (second) generation leptoquarks up to $1050 \mathrm{GeV}(1000 \mathrm{GeV})$.

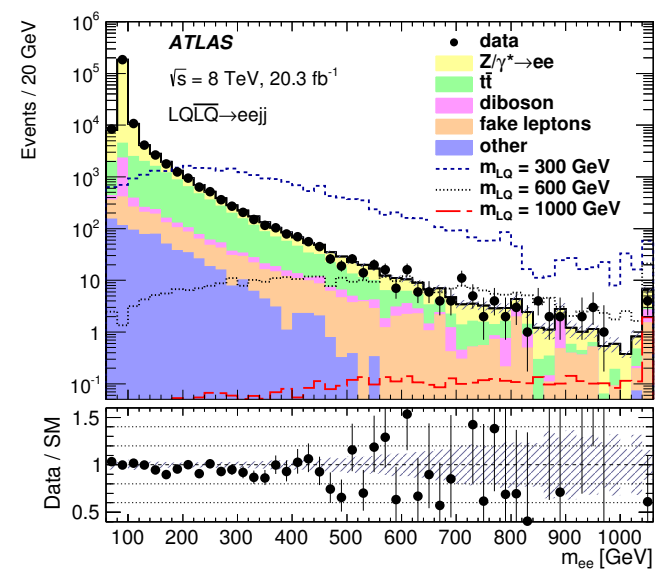

(a)

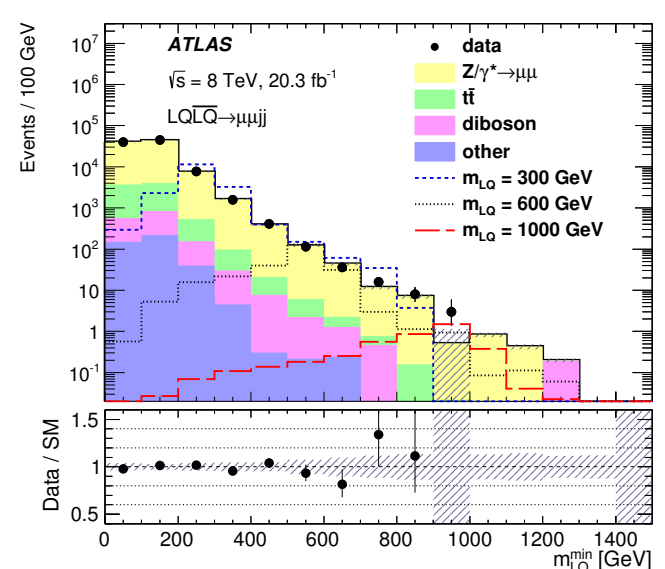

(b)

Figure 9: Distribution of $m_{\mathrm{ee}}$ (a) and $m_{\mathrm{LQ}}^{\min }$ in the muon channel. Both diagrams from [26]

\section{First results from LHC Run 2}

First results in $78 \mathrm{pb}^{-1}$ of LHC Run 2 data were presented in the dilepton final state and in the diphoton final state $[28,29]$. These first results follow the strategies of analyses described in sections 2 and 3. With this small data sample it is not expected that either analysis is sensitive to new physics. The dielectron, dimuon and diphoton invariant mass distributions are shown in Fig. 10.

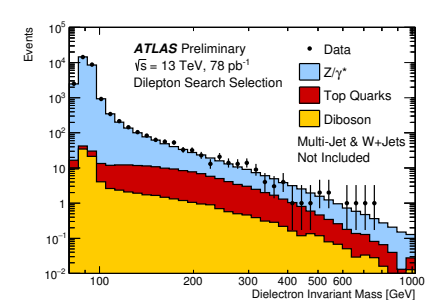

(a)

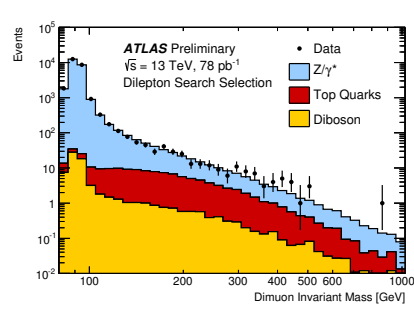

(b)

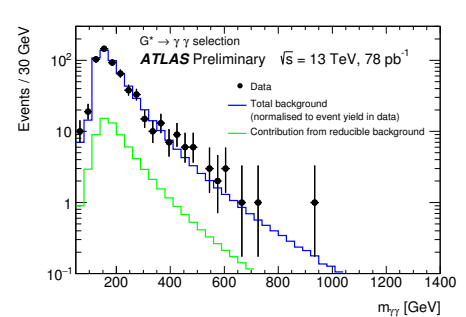

(c)

Figure 10: Distribution of dielectron system invariant mass (a) and dimuon invariant mass (b) from [28] and diphoton invariant mass from [29] (c).

\section{Conclusions}

In this proceedings several searches for new physics at the ATLAS experiment were presented using the full LHC Run 1 dataset. A large variety of final states were explored. No new physics was observed and several new physics models were constrained up to mass scales of several $\mathrm{TeV}$. 
In addition a first look at the LHC Run 2 ATLAS data were shown in the cleanest final states - the dilepton and the diphoton signatures.

\section{References}

[1] ATLAS Collaboration The ATLAS Experiment at the CERN Large Hadron Collider, JINST 3 (2008) S08003

[2] ATLAS Collaboration Search for High-Mass Dilepton Resonances in pp Collisions at $\sqrt{s}=8 \mathrm{TeV}$ with the ATLAS Detector, Phys. Rev. D 90, 052005 (2014) [hep-ex/1405.4123]

[3] ATLAS Collaboration Search for contact interactions and large extra dimensions in the dilepton channel using proton-proton collisions at $\sqrt{s}=8 \mathrm{TeV}$ with the ATLAS detector, Eur. Phys. J. C 74, 3134 (2014) [hep-ex/1407.2410]

[4] P. Langacker The Physics of Heavy Z' Gauge Bosons, Rev. Mod. Phys. 811199 (2009) [hep-ph/0801.1345]

[5] D. London and J. L. Rosner Extra Gauge Bosons in E(6), Phys. Rev. D 341530 (1986)

[6] L. Randall and R. Sundrum A Large Mass Hierarchy from a Small Extra Dimension, Phys. Rev. Lett. 833370 (1999) [hep-ph/9905221].

[7] E. Salvioni, G. Villadoro, and F. Zwirner Minimal Z' models: present bounds and early LHC reach JHEP 11068 (2009) [hep-ph/0909.1320]

[8] E. Eichten, K.D. Lane, M.E. Peskin New Tests for Quark and Lepton Substructure Phys. Rev. Lett. 50 811 (1983)

[9] E. Eichten, I. Hinchliffe, K.D. Lane, C. Quigg Supercollider physics Rev.Mod. Phys. 56579 (1984)

[10] N. Arkani-Hamed, S. Dimopoulos, G. Dvali The Hierarchy Problem and New Dimensions at a Millimeter Phys. Lett. B 429263 (1998). [hep-ph/9803315]

[11] ATLAS Collaboration Search for high-mass diphoton resonances in pp collisions at $\sqrt{s}=8 \mathrm{TeV}$ with the ATLAS detector Phys. Rev. D 92032004 (2015) [hep-ex/1504.05511]

[12] ATLAS Collaboraion Search for new particles in events with one lepton and missing transverse momentum in pp collisions at $\sqrt{s}=8 \mathrm{TeV}$ with the ATLAS detector JHEP 09 (2014) 037 [hep-ex/1407.7494]

[13] G. Altarelli, B. Mele, and M. Ruiz-Altaba Searching for new heavy vector bosons in pp colliders Z. Phys. C 45109 (1989)

[14] ATLAS Collaboration Search for new phenomena in the dijet mass distribution using pp collision data at $\sqrt{s}=8$ TeV with the ATLAS detector Phys. Rev. D. 91052007 (2015) [hep-ex/1407.1376]

[15] ATLAS Collaboration Search for new phenomena in dijet angular distributions in proton-proton collisions at $\sqrt{s}=8$ TeV measured with the ATLAS Detector Phys. Rev. Lett. 114221802 [hep-ex/1504.00357]

[16] U. Baur, I. Hinchliffe, D. Zeppenfeld Excited Quark Production at Hadron Colliders Int. J. Mod.Phys. A 21285 (1987)

[17] U. Baur, M. Spira, P. M. Zerwas Excited-quark and-lepton production at hadron colliders Phys. Rev. D 42815 (1990) 
[18] L. A. Anchordoqui, J. L. Feng, H. Goldberg, A. D. Shapere Inelastic Black Hole Production and Large Extra Dimensions Phys. Lett. B 594363 (2004) [hep-ph/0311365]

[19] P. Meade and L. Randall Black Holes and Quantum Gravity at the LHC JHEP 05003 (2008) [hep-ph/0708.3017]

[20] X. Calmet, W. Gong, and S. D. Hsu Colorful quantum black holes at the LHC Phys. Lett. B 66820 (2008) [hep-ph/0806.4605]

[21] D. M. Gingrich Quantum black holes with charge, colour, and spin at the LHC J. Phys. G 37105008 (2010) [hep-ph/0912.0826]

[22] ATLAS Collaboration Search for new phenomena in photon+jet events collected in protonâĂŞproton collisions at $\sqrt{s}=8 \mathrm{TeV}$ with the ATLAS detector Phys. Lett. B 728C 562-578 (2014) [hep-ex/1309.3230]

[23] S. Dimopoulos, R. Emparan String balls at the LHC and beyond Phys. Lett. B $526393 a ̂ A ̆ S ̧ 398$ (2002) [hep-ph/0108060]

[24] ATLAS Collaboration Search for low-scale gravity signatures in multi-jet final states with the ATLAS detector at $\sqrt{s}=8 \mathrm{TeV}$ JHEP 07032 (2015) [hep-ex/1503.08988]

[25] ATLAS Collaboration A search for high-mass resonances decaying to $\tau^{+} \tau^{-}$in pp collisions at $\sqrt{s}=8$ TeV with the ATLAS detector JHEP 07157 (2015) [hep-ex/1502.07177]

[26] ATLAS Collaboration Searches for scalar leptoquarks in pp collisions at $\sqrt{s}=8$ TeV with the ATLAS detector [hep-ex/1508.04735]

[27] W. Buchmuller, R. Ruckl, and D. Wyler Leptoquarks in lepton-quark collisions Phys. Lett. B 191442 (1987).

[28] ATLAS Collaboration Public plots from the ATLAS dilepton search at $13 \mathrm{TeV}$ https://atlas.web.cern.ch/Atlas/GROUPS/PHYSICS/PLOTS/EXOT-2015-001/

[29] ATLAS Collaboration Diphoton invariant mass spectrum in early Run 2 data collected by the ATLAS detector at $\sqrt{s}=13 \mathrm{TeV}$ https://atlas.web.cern.ch/Atlas/GROUPS/PHYSICS/PLOTS/EXOT-2015-003/ 